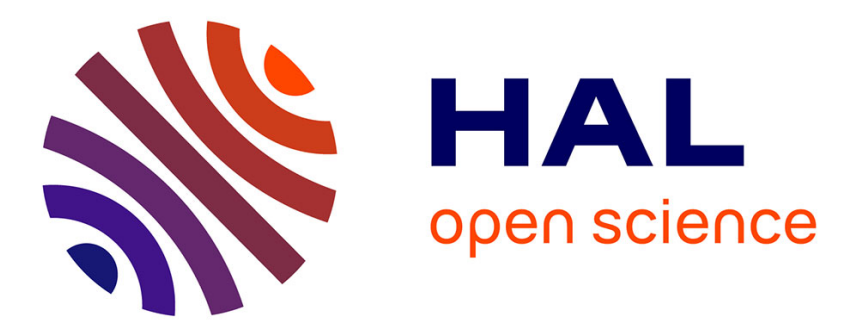

\title{
Optical detection of cold atoms without spontaneous emission
}

\author{
Véronique Savalli, Gabriel Zs. Horvath, Paul D. Featonby, Laurent Cognet, \\ Nathalie Westbrook, Christoph I Westbrook, Alain Aspect
}

\section{- To cite this version:}

Véronique Savalli, Gabriel Zs. Horvath, Paul D. Featonby, Laurent Cognet, Nathalie Westbrook, et al. Optical detection of cold atoms without spontaneous emission. Optics Letters, 1999, 24 (22), pp.1552-1554. 10.1364/OL.24.001552 . hal-00872120

HAL Id: hal-00872120

https://hal-iogs.archives-ouvertes.fr/hal-00872120

Submitted on 11 Oct 2013

HAL is a multi-disciplinary open access archive for the deposit and dissemination of scientific research documents, whether they are published or not. The documents may come from teaching and research institutions in France or abroad, or from public or private research centers.
L'archive ouverte pluridisciplinaire HAL, est destinée au dépôt et à la diffusion de documents scientifiques de niveau recherche, publiés ou non, émanant des établissements d'enseignement et de recherche français ou étrangers, des laboratoires publics ou privés. 


\title{
Optical detection of cold atoms without spontaneous emission
}

\author{
V. Savalli, G. Zs. K. Horvath, P. D. Featonby, L. Cognet, N. Westbrook, C. I. Westbrook, and A. Aspect \\ Laboratoire Charles Fabry de l'Institut d'Optique, Unité Mixte du Centre National de la Recherche Scientifique 8501, \\ B.P. 147, 91403 Orsay Cedex, France
}

Received July 12, 1999

\begin{abstract}
We have demonstrated nondestructive detection of cold atoms with a probe laser by a frequency-modulation spectroscopy technique. We were able to tune the probe laser and its sidebands far from atomic resonance to reduce the spontaneous emission to less than 0.2 photon per atom during detection. (C) 1999 Optical Society of America

OCIS codes: $\quad 300.6380,020.7010$.
\end{abstract}

The ability to monitor an ensemble of cold atoms optically without significantly perturbing it is of great value to the atomic-optics research community. Experiments have already been reported in which a single Bose-Einstein condensate was nondestructively monitored with an imaging system over a large number of exposures. ${ }^{1} \quad$ With such detection one can follow a single sample through time and avoid noise from fluctuating initial conditions. A nondestructive time-offlight measurement of multiple bounces on an atomic mirror would, for example, be particularly useful. ${ }^{2}$ In this Letter we report the development of such a detection method that uses frequency modulation (FM) spectroscopy.

By nondestructive we mean with little spontaneous emission, because spontaneous emission is often the main process that destroys the coherence of an atomic sample. ${ }^{3}$ Therefore we seek to increase the detuning of the probe laser beam from resonance to reduce the spontaneous scattering rate and detect the dispersion, or the phase shift on a laser beam that is due to the atoms. ${ }^{4}$ A uniform sample of two-level atoms shifts the optical phase of a weak laser beam by

$$
\varphi(\Delta)=-\frac{3 \lambda^{2}}{2 \pi} \frac{\Gamma \Delta}{4 \Delta^{2}+(\Gamma)^{2}} \rho l=-\frac{\Delta}{\Gamma} \frac{l}{l_{\mathrm{MFP}}},
$$

where $\Delta$ is the detuning from atomic resonance (defined by the frequency of the laser minus the frequency of the atomic transition), $\lambda$ is the laser wavelength, $\Gamma$ is the natural linewidth of the atomic transition, $l$ is the thickness of the sample, $l_{\mathrm{MFP}}$ is the mean free path of a photon associated with near-resonant scattering, and $\rho$ is the density of the atomic cloud.

In the ideal case, the detection of this phase shift is limited by the shot noise of the probe laser; thus the signal-to-noise ratio, $\mathrm{S} / \mathrm{N}=\varphi / \delta \varphi$, varies as $\sqrt{I} / \Delta$, where $I$ is the probe laser intensity. The mean number of spontaneously scattered photons $N_{\mathrm{sp}}$ varies as $I / \Delta^{2}$; thus, fixing $N_{\text {sp }}$ fixes the $\mathrm{S} / \mathrm{N}$ in the shot-noise limit. This point was made in Ref. 5: Assuming that dispersive effects dominate, $\Delta \gg \Gamma / 2$, there is nothing to be gained by using large detunings if one fixes $N_{\mathrm{sp}}$.

Real detection systems are, of course, often limited by other sources of noise, and the optimization of the detuning, for example, is more complex. Here we describe a system in which we employ FM spectroscopy ${ }^{6}$ to make time-of-flight measurements by using the phase shift induced by cold atoms on a laser beam. FM spectroscopy is a well-established technique that has been used in particular as a high-sensitivity detector of trace molecules in gas cells. ${ }^{7}$ In these experiments one generally uses high enough laser power that an important noise limitation is the noise in the residual amplitude modulation that accompanies the FM. Here we used powers so low that the dark noise of the detector was also a significant factor. We sought to decrease $N_{\mathrm{sp}}$ to fewer than one per atom. For example, $N_{\mathrm{sp}}=0.1$ means that $9 / 10$ of the atoms do not scatter any light and are in an unperturbed state for a second probe pulse.

To generate the FM sidebands for our detection system we modulate the injection current of a laser diode at frequency $\Omega$. Following Ref. 7, the resultant electric field is well approximated by

$$
\begin{aligned}
E(t)= & E_{0} \exp \left(-i \omega_{L} t\right)[1+M \sin (\Omega t+\psi)] \\
& \times \exp (i \beta \sin \Omega t)+\text { c.c. },
\end{aligned}
$$

where $\omega_{L}$ is the carrier frequency, $M$ and $\beta$ are the modulation indices for the amplitude modulation (AM) and the FM, respectively, and $\psi$ is the relative phase between the two types of modulation. Inasmuch as the AM and FM modulation indices are relatively weak in our case, we assume that the field has only three spectral components: the carrier and two sidebands. When the atoms interact with the field, the three frequency components experience different phase shifts, and the beat note between the carrier and the sidebands at the modulation frequency $\Omega$ is modified.

We observe the beat note on a photodiode. This beat note is mixed with a local oscillator at frequency $\Omega$ and with a relative phase $\theta$. After a low-pass filter the resultant photocurrent is (to first order in the phase shift)

$$
\begin{aligned}
i= & K\left[M J_{0}(\beta) \cos (\theta-\psi)+\frac{M J_{0}(\beta)}{2}\left(\varphi_{+}-\varphi_{-}\right)\right. \\
& \left.\times \sin (\theta-\psi)-J_{1}(\beta)\left(\varphi_{-}+\varphi_{+}-2 \varphi_{0}\right) \cos (\theta)\right] .
\end{aligned}
$$


$K$ is a constant that depends on the incident light intensity, the quantum efficiency of the photodiode, and the gain of the electronics. The quantities $\varphi_{-}, \varphi_{+}$, and $\varphi_{0}$ are the phase shifts given by Eq. (1) for the lower and upper sidebands and the carrier, respectively. The first term in Eq. (3) corresponds to the residual amplitude modulation of the laser diode, and it is independent of the presence of the atoms. If one chooses the phase of the local oscillator such that $\theta-\psi=(2 n+1) \pi / 2$, where $n$ is an integer, the residual AM term can be suppressed, and hence noise induced by this term is avoided. Both the second and the third terms in Eq. (3) can be used to detect the presence of the atoms. In our situation the third term is approximately four times larger than the second term.

In our experiment, the modulation frequency $\Omega / 2 \pi$ was equal to $800 \mathrm{MHz}$. The probe laser beam for the nondestructive detection came from a free-running Hitachi laser diode at $780 \mathrm{~nm}$. We measured a beat note between this beam and an auxiliary laser locked to a $\mathrm{Rb}$ transition to determine the absolute frequency of the probe. By measuring the AM on the laser beam at $800 \mathrm{MHz}$ and by observing the optical spectrum of the modulated laser diode with a Fabry-Perot interferometer, we found the modulation parameters to be $M \approx 0.1, \beta \approx 0.6$, and $\psi \approx 1.1 \pi$. The electronic circuit used for the detection is shown in Fig. 1. To choose the relative phase $\theta$ and thus suppress the first term of Eq. (3) we mounted the fast photodiode (New Focus Model 1601) upon a translation stage. With $\varphi=0$ (no atoms) we suppressed the first term by translating the photodiode and thus adjusting the phase of the signal at the frequency $\Omega$ before the mixer. The noise after the low-pass filter was observed as a function of incident laser power. For optical powers greater than $100 \mu \mathrm{W}$, the rms amplitude noise increased linearly with the laser power, indicating that it was due to laser technical noise. Below $100 \mu \mathrm{W}$, the limitation was the noise of the detector.

To optimize the $\mathrm{S} / \mathrm{N}$ and minimize $N_{\mathrm{sp}}$ one must carefully choose the values of the probe laser intensity $I$ and the detuning $\Delta$ of the carrier from the atomic resonance. Small detunings are clearly undesirable because they require small intensities and we are limited by the intensity-independent detector noise. Detunings that are large compared with $\Omega$ are also undesirable because the signal in Eq. (3) decreases faster than $1 / \Delta$ for such detunings. A calculation assuming a detector-noise-limited measurement and taking into account our relative sideband intensities and the transition strengths for an ${ }^{85} \mathrm{Rb}$ atom showed that the optimum detuning has a broad maximum near $\Delta / 2 \pi=500 \mathrm{MHz}$. In practice we used a detuning of $400 \mathrm{MHz}$.

The experimental setup was the same as that used in Ref. 8. Approximately $2 \times 10^{7}{ }^{85} \mathrm{Rb}$ atoms were loaded into a magneto-optical trap (MOT) every $2.4 \mathrm{~s}$. The atoms were prepared in the $F=3$ level of the $5 S_{1 / 2}$ state and released from the MOT. The probe beam for the nondestructive detection, which we call the dispersive probe, was located $5.2 \mathrm{~mm}$ below the MOT and was linearly polarized. The carrier frequency of this beam was detuned above the atomic resonance $\left(5 S_{1 / 2} F=3 \rightarrow 5 P_{3 / 2} F=4\right)$ by $400 \mathrm{MHz}$. The laser beam had a FWHM of $2.5 \mathrm{~mm}$ horizontal and $1.9 \mathrm{~mm}$ vertical.

Curve (a) of Fig. 2 shows a typical nondestructive time-of-flight signal. The signal was acquired for a power of $4 \mu \mathrm{W}$ in the probe beam. The single-shot $\mathrm{S} / \mathrm{N}$ was approximately unity. The absolute size of the detected signal was consistent with the measured number of atoms and our measured overall gain to within better than a factor of 2 . To calculate the signal theoretically we took into account the Gaussian shape of the cloud at the probe ( $\mathrm{rms}$ size, $\sigma=1.4 \mathrm{~mm}$ ), that of the probe beam, and the hyperfine structure of the $\mathrm{Rb}$ atom.

To measure the value of $N_{\mathrm{sp}}$ we compared the results with those for another probe beam, which we call the destructive probe, whose detuning was small. This probe was located $11.6 \mathrm{~mm}$ below the MOT and was detuned $5 \mathrm{MHz}$ below the $5 S_{1 / 2} F=3 \rightarrow 5 P_{3 / 2} F=4$

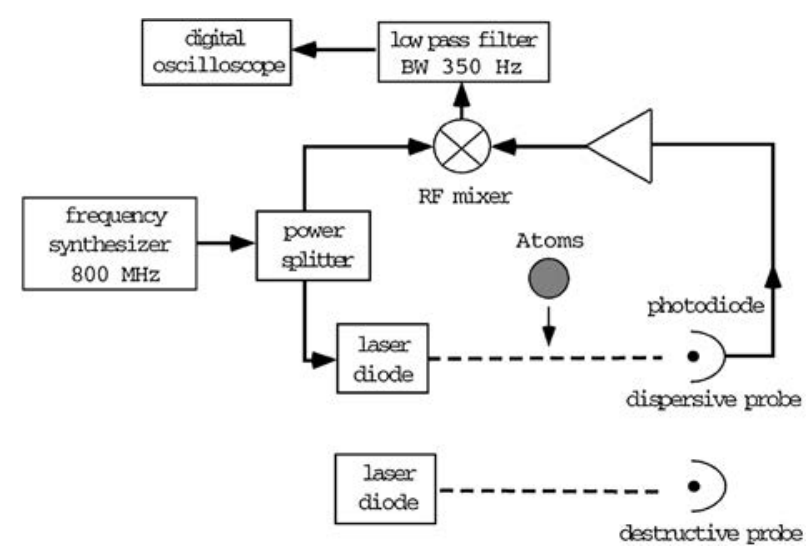

Fig. 1. Schematic diagram of the experiment. A cloud of cold atoms fell through a far detuned probe laser beam. The FM spectroscopy setup shown was sensitive to the phase shift induced by the atoms on the laser beam. To monitor the effect of the dispersive probe, we placed a second probe beam below the first.

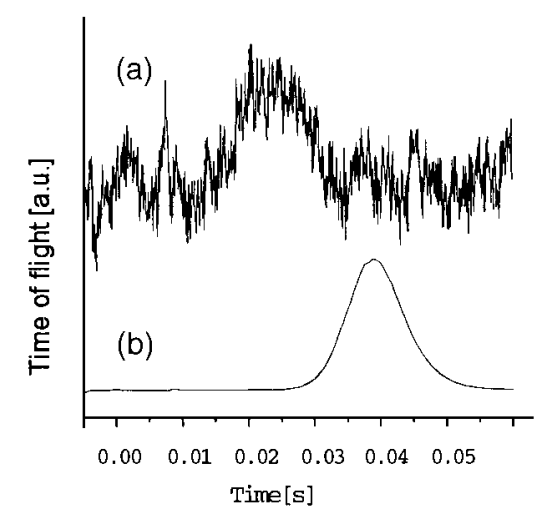

Fig. 2. Typical time-of-flight signals after an average of 20 shots. (a) The nondestructive signal. The power in the dispersive probe was $4 \mu \mathrm{W}$ and, during this detection, less than 0.2 photon was scattered per atom. (b) The signal obtained with a destructive probe located below the dispersive probe. The two curves were taken simultaneously with both probes present. 


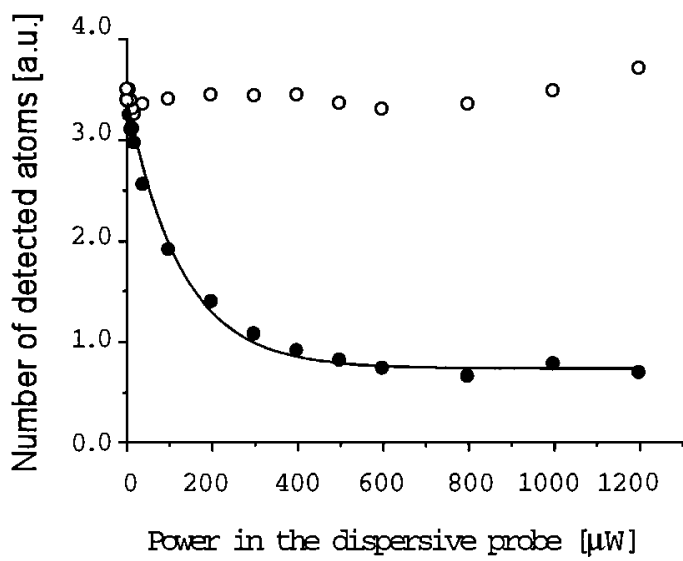

Fig. 3. Number of atoms detected with the destructive probe as a function of the optical power in the dispersive probe beam (filled circles). Open circles, the data when the dispersive probe was blocked, showing the fluctuations of the number of atoms detected by the destructive probe. The curve is a fit to an exponential decay plus a background. This background is due to the fact that all the atoms did not pass through the dispersive probe, because of the relative sizes of the atomic cloud and the beam.

transition. With it, we detected the atoms by absorption just after their interaction with the dispersive probe beam. The destructive probe could be superposed with a repumping beam so that either all the atoms or only those of the $5 S_{1 / 2} F=3$ level were detected. When the repumper was present, the destructive signal was independent of the power in the dispersive probe. This was not the case when no repumper was present. In the latter situation we could thus study the hyperfine pumping of the atoms by the dispersive probe. Curve (b) of Fig. 2 is the destructive signal. During the destructive detection approximately 500 photons were scattered per atom. Figure 3 shows the number of atoms detected with the destructive probe as a function of the laser power in the dispersive probe beam. By comparing the destructive signals with and without the dispersive probe, we could deduce the rate at which atoms made a transition from the $F=3$ level to the $F=2$ level during the interaction with the dispersive beam. A calculation of the branching ratios that took into account the two sidebands and assumed that the Zeeman sublevels in the $F=3$ level were equally populated indicated that only one scattered photon in six resulted in a transition from the $F=3$ level to the $F=2$ level. From the curve in Fig. 3 and this calculation, we deduced that
$N_{\text {sp }} \approx 0.2$ at $4 \mu \mathrm{W}$. This value is in agreement with a theoretical calculation of the number of scattered photons.

It is interesting to compare our observed $\mathrm{S} / \mathrm{N}$ with the shot-noise limit. With a quantum efficiency of $50 \%$, a detection bandwidth of $350 \mathrm{~Hz}$, and the same values for $\beta$ and $M$ as in the experiment, we found that for shot-noise-limited detection $\mathrm{S} / \mathrm{N} \approx 400 \times \sqrt{P}$, where $P$ is the laser power in milliwatts. At $P=$ $4 \mu \mathrm{W}$ we found that the shot-noise-limited $\mathrm{S} / \mathrm{N}$ is approximately 25 . The limit was the noise of the detector, and we found a level consistent with that quoted by the manufacturer.

It is natural to consider how to improve this method. The use of a lower-noise detector, such as a photomultiplier tube, should easily render the detector noise negligible if the stray light can be shielded sufficiently. In our experiment the typical detected atomic density was $\sim 5 \times 10^{8} \mathrm{~cm}^{-3}$. Bose-Einstein condensates, on the other hand, can have densities 4 orders of magnitude larger. The detection that we have described here would therefore easily be able to monitor a condensate that bounces several times on an atomic mirror.

This research was supported by the Région Ile de France and the European Union TMR network (contract ERBFMRXCT960002). V. Savalli's e-mail address is veronique.savalli@iota.u-psud.fr.

\section{References}

1. M. R. Andrews, M.-O. Mewes, N. J. van Druten, D. S. Durfee, D. M. Kurn, and W. Ketterle, Science 273, 84 (1996). C. C. Bradley, C. A. Sackett, and R. G. Hulet, Phys. Rev. Lett. 78, 985 (1997).

2. C. G. Aminoff, A. M. Steane, P. Bouyer, P. Desbiolles, J. Dalibard, and C. Cohen-Tannoudji, Phys. Rev. Lett. 71, 3083 (1993). C. V. Saba, P. A. Barton, M. G. Boshier, I. G. Hughes, P. Rosenbusch, B. E. Sauer, and E. A. Hinds, Phys. Rev. Lett. 82, 468 (1999).

3. P. Berman, ed., Atom Interferometry (Academic, San Diego, Calif., 1997).

4. J.-Y. Courtois, J.-M. Courty, and S. Reynaud, Phys. Rev. A 52, 1507 (1995); A. Aspect, R. Kaiser, N. Vansteenkiste, P. Vignolo, and C. I. Westbrook, Phys. Rev. A 52, 4704 (1995).

5. S. L. Rolston and W. D. Phillips, "Imaging BoseEinstein condensates: How destructive is nondestructive imaging?" submitted to Phys. Rev. A.

6. G. C. Bjorklund, Opt. Lett. 5, 15 (1980).

7. J. A. Silver, Appl. Opt. 31, 707 (1992).

8. A. Landragin, G. Labeyrie, C. Henkel, R. Kaiser, N. Vansteenkiste, C. I. Westbrook, and A. Aspect, Opt. Lett. 21, 1595 (1996). 